\title{
Motivation and the Organization of Behaviour
}

\author{
G. MASON AND M. BATESON
}

\subsection{What is Motivation and Why is it Important?}

\author{
Motivation as a 'causal' explanation for animal behaviour
}

Motivational states include thirst, hunger, fear and the urges to migrate, mate, nest-build and dust bathe. These motivations are internal states which vary in magnitude from one moment to the next, and that help determine which stimuli animals will react to and which they will not; which goals they seek out and which they do not; and the effort or intensity with which they will perform a given behaviour. Motivations are examples of causal explanations for behaviour (see Chapter 1), meaning that they are a proximate, mechanistic explanation for why an animal is currently performing a particular behaviour pattern.

Not all changes in behaviour are due to changes in motivation. We do not invoke motivation to explain behaviours that are always elicited in the same way by the same stimuli (e.g. reflexes like blinking, or rapid limb withdrawal from painful stimuli); changes in the cues that animals respond to as a result of learning (see Chapter 5); nor many of the behavioural changes that result from developmental processes (e.g. maturation) or illness/injury (e.g. ceasing to eat because of a gum abscess). Instead, the properties of animal behaviour that motivation helps explain can be summarized as follows. First, changes in motivational state help explain the decisions made by individual animals when faced with choices about what activity to do next. Thus, if you watch an animal for any period you will notice that it engages in different behaviour patterns, apparently spontaneously switching between these at intervals.

For example, in the middle of the day, domestic chickens switch from foraging to dust bathing; while a domestic cat during the course of its day will switch from feeding to drinking, from hunting to resting, and so on. Changes in motivational state help explain why sometimes external stimuli act as powerful triggers for behaviour, but at other times are effectively ignored. For instance, if a hen is deprived of a suitable substrate in which to dust bathe, she will dust bathe vigorously when she is finally allowed access to, say, wood shavings or peat. However, once she has finished doing this, for a while afterwards that very same substrate will lose its ability to stimulate her to perform any further dust bathing. Finally, when watching our deprived hen indulge in dust bathing, we might well notice an increase in this activity's duration and intensity. Thus, along with the increased probability of occurrence of a specific behaviour pattern, we typically see other changes as well, such as increased rates of performance and, if obstacles are placed in an animal's way, increased efforts made to perform the behaviour.

\section{Motivational states as intervening variables}

From the section above, and perhaps from our own personal experiences, we can see that strong motivations alter behaviour in many different ways. For example, if we 
look at drinking behaviour we notice that, as the motivational state of thirst increases, the probability that drinking will occur also increases; but also the range of liquids found acceptable increases (for instance, rats will tolerate quinine in water if they are thirsty - a bitter substance they otherwise avoid), the latency to start drinking decreases (i.e. it is initiated more rapidly when liquid is presented) and the rate of drinking and the amount consumed both increase. If the animal has to pay a cost to drink, it will also be more willing to do so, and this might involve learning novel responses: thus, just as hot and thirsty humans will pay inflated prices for cold sodas and bottled water, so will thirsty rats press a lever multiple times to gain water. As we discuss later, one reason for animals being prepared to perform effortful responses is that being unable to perform highly motivated behaviours is frustrating and accompanied by negative emotions, whereas performing them is accompanied by positive ones. Thus, serious thirst is subjectively unpleasant, and water also tastes wonderful when we are thirsty (see, e.g. Rolls, 2005).

The suite of behavioural and emotional changes described above is, in turn, induced by a suite of diverse events or 'inputs' originating from physiological signals from the body (e.g. hormonal changes, signals of homeostatic need) and sensory inputs from the external world. For instance, the motivation to drink can be elicited by just having eaten, by a dry mouth, by hypernatraemia (high blood sodium levels), by the sight of another animal drinking, by the smell of a very palatable drink or by the sight or sound of other external cues that, through learning (see Chapter 5) have come to be associated with drinking.

If we were to draw all these various possible causal links outlined above, we would come up with a very complicated diagram (see Fig. 4.1a). A far simpler, more economical way of summarizing the many observed relationships is instead to postulate a single 'intervening variable' - in this case something called 'thirst' - that is influenced by all the many independent variables (dry mouth, external cues and so on) and in turn influences all the dependent variables (amount consumed, perceived pleasantness of

(a)

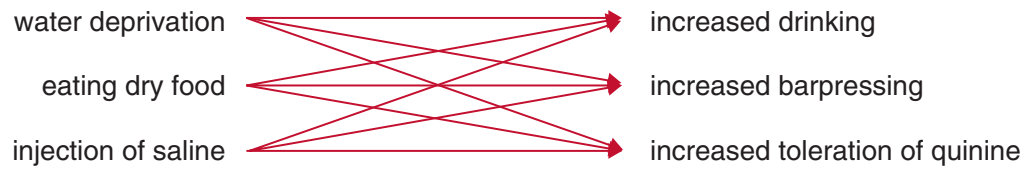

(b)

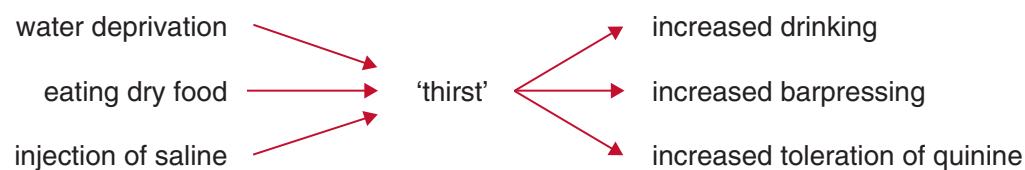

Fig. 4.1. Two alternative models for the explanation of behaviour. Three causal factors on the left (water deprivation, ingestion of dry food and injection of saline) lead to three types of response on the right (increased drinking, increased bar pressing for water and increased toleration of quinine in available drinking water). Miller noted that explanations could involve postulating nine stimulus-response links (a) or a smaller number of links if an intervening variable ('thirst') is included (b) (after Miller, 1956 in Barnard, 2004). 
drinking and so on; see Fig. 4.1b). Motivational states are thus best conceptualized as intervening variables that provide an integrative link between various functionally related inputs and behavioural outputs. This general concept underlies the more complex models of motivation described in this chapter. But first, how and where does this 'integrative link' occur?

\section{Where and how do motivational states occur?}

Since it is the brain that drives and controls behaviour, it is a truism that motivational states arise in the brain. Indeed, a 'motivational state' can be defined as the combined physiological and perceptual state of an animal, as represented in its brain. Beyond acknowledging this, however, ethologists have traditionally treated the animal as a 'black box', meaning that they are not interested in the details of the mechanisms inside it. Under this approach, the focus is more on understanding the 'rules' that translate the current internal state of the animal and the external stimuli to which it is exposed in observed behaviour. This 'black box' approach can be useful because it can help us to model, quite simply, how it is possible for the same cues to elicit different responses at different times and in different individuals. To illustrate this, if we understand how dust bathing is affected by the oiliness of feathers and the length of deprivation from a bathing substrate, we can then understand why some birds might have higher motivation to dust bathe than others when presented with an appropriate substrate. Likewise, if we can record how animals weigh up the costs and benefits of performing particular behaviours (e.g. gaining water to drink versus expending time and energy lever pressing for it) we can model and predict many aspects of observed behavioural decision making. Knowing the exact internal physiological mechanisms by which these processes occur would not necessarily yield information with any more explanatory power.

However, in other instances, understanding the precise physiological mechanisms giving rise to motivational states can be very useful; opening the black box to dissect its endocrine and neural mechanisms may give us unique insights. In his work on periparturient sows, for example, Gilbert has shown that these animals' nesting motivations are responses elicited within the brain by prostaglandins that are released as part of physiological preparation for birth. By showing exactly how motivations to perform this specific natural behaviour arise, this mechanistic research reveals how unlikely it is that we will be able to reduce this motivation by any intervention other than by allowing sows actually to build nests (see, e.g. Boulton et al., 1997).

Neurobiologists have also recently become interested in the mechanisms underlying motivational states. This exciting area of research is fuelled, not by a desire to understand animal behaviour, but instead by the need to understand problematic 'over-motivated' behaviours in humans: compulsions and addictions such as overeating, gambling and drug taking. It is beyond the scope of this chapter to review the neurobiology of motivation. However, in brief, forebrain regions such as the orbitofrontal cortex and the basal ganglia's nucleus accumbens and have been found to control the effort and intensity with which motivationally relevant stimuli are sought out and responded to, and have also been implicated in addiction and in abnormal compulsive behaviours with an emotional component. Berridge (2004) and Rolls (2005) provide good further reading for those wishing more detail. Applied ethologists are now starting to find this mechanistic information useful for investigating 
abnormal behaviour in captive animals (see below and Chapter 7). Some parallel work by psychologists on both animal and human subjects has also helped clarify the links between motivation, emotion and learning - something with important animal welfare implications, as we discuss below.

\section{Why it is important to understand domestic animals' motivations}

Understanding animal welfare and the origins of abnormal behaviour are two of the main reasons why it is important to investigate domestic animals' motivations. Motivational research helps us identify - and hopefully alleviate - the sources of some important frustration-related welfare problems; it also helps reveal why abnormal activities like stereotypic behaviours are so common in captive animals. However, even if we are not interested in welfare, understanding motivation can still be important practically, because it can aid the effective management of both learnt and nonlearnt aspects of behaviour. For example, if we appreciate how both the nature of internal states and the quality of external cues affects motivation, we can use this to design training regimes where the rewards offered are most likely to cause animals to learn what we want them to learn. We also can use this understanding to manipulate instinctive aspects of behaviour such as mating and maternal care. We expand on all four of these topics in Section 4.4, below.

\subsection{Motivational States and the Organization of Behaviour}

\section{The interaction of internal and external stimuli}

Motivational states can be induced by internal stimuli (signals from the body), external stimuli (cues in the outside world) and the interactions between them (i.e. how they combine to influence the animal).

Perhaps the most obvious type of internal stimulus to affect motivation is one that reflects a disruption of homeostasis, leading to motivations to perform behaviours that will restore an animal's physiological equilibrium. Thus cues from the body signalling dehydration induce strong motivation to drink, while cues from the body signalling low energy induce motivation to eat. Other common examples of internal states important for motivation include reproductive hormones. For example, in terms of performing operant responses like lever pressing, or overcoming aversive barriers like electrified floor-grids, female rhesus monkeys will work harder to reach a male when they are in oestrus than when they are not. Female marmosets will also lever press for the sight and sounds of an infant, but only when they are heavily pregnant or lactating - an effect mediated by oestrogen. The role of hormones in the nest-building motivation of sows has already been touched upon, and is developed further in Chapter 3.

External stimuli that affect motivation include signals of time of day or seasons of the year (the latter often exerting their effects via endocrine changes), and cues that signal a motivationally relevant resource. These are sometimes known as 'eliciting stimuli'. We are all familiar with the way the smell of a delicious meal can make us feel ravenously hungry. Eliciting cues can likewise have powerful effects on animals' motivations, in behavioural systems as diverse as feeding and mating. They are particularly 
important in triggering escape motivations (elicited by predator cues), aggressive motivations (elicited by cues from rival conspecifics) and, in cats and other carnivores, predatory responses like pouncing and biting (elicited by the movements, squeaks and struggles of prey). Sometimes animals will habituate to one type of cue, only to find a new one powerfully motivating: thus the presentation of new sexual partners, or of new types or flavours of food, may boost or renew mating or feeding motivations (a phenomenon known as the Coolidge effect, after the American president of the same name). Note that learnt cues (e.g. the sight of a favourite restaurant to humans; or sound of the fridge door opening to a cat) can affect motivational states too.

Most motivated behaviours are affected by a combination of external cues and internal states. Classic work by Baerends et al. (1955, described in McFarland, 1985) on courtship behaviour in male guppies demonstrates how the size of the female fish (an external stimulus that relates to her attractiveness) and the colour patterns on a male (an external indication of the male's internal sexual state) combine to affect his behaviour. Male guppy courtship proceeds through a series of stages from posturing, via intentional sigmoid displays to full sigmoid displays. Figure 4.2 shows that the attractiveness (i.e. size) of the female required to elicit a given display declines as the internal mating motivation of the male increases. The principles revealed in this experiment apply to many other behaviour systems. For example, food-deprived animals need only poor-quality cues to elicit feeding, while sated animals will respond only to eliciting stimuli that signal highly preferred, palatable or novel food items.

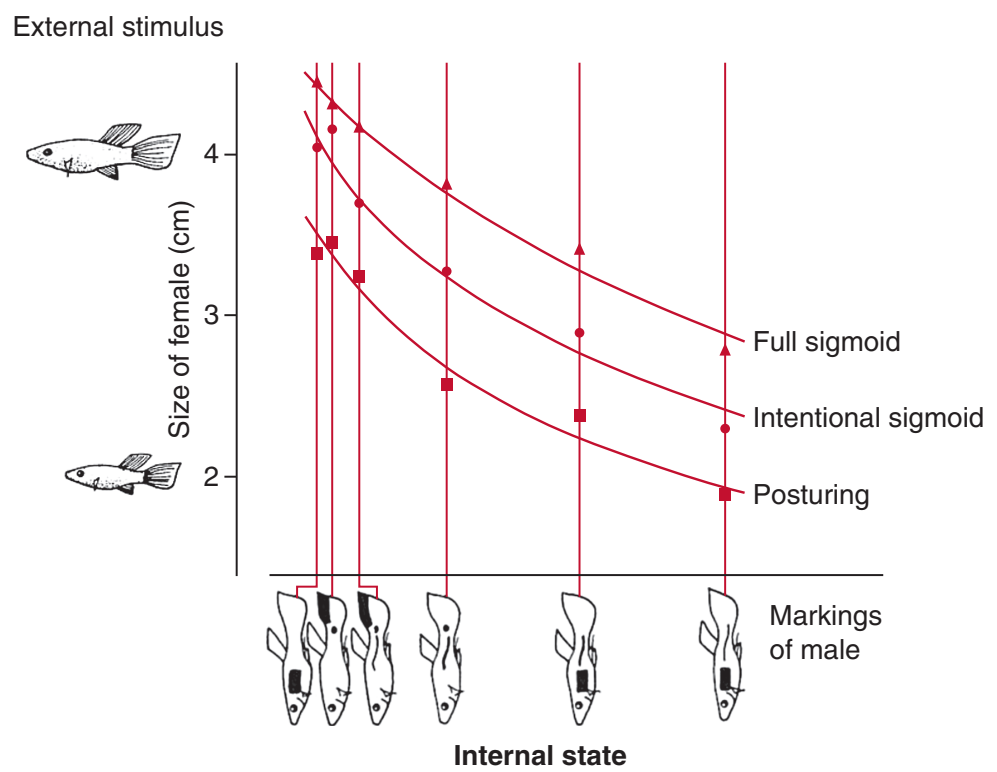

Fig. 4.2. The size of a female guppy needed to elicit each of three different displays (posturing, intentional sigmoid and full sigmoid) from male guppies as a function of the markings on the male. The size of the female is an external stimulus and the markings on the male are an indication of his internal state. The smooth lines plot 'motivational isoclines' lines that connect points of equal likelihood that a male will perform a given display (modified from Baerends et al., 1955 in McFarland, 1985). 
The shape of the motivational isoclines in Fig. 4.2 may tempt us into thinking that female attractiveness and male sexual motivation can be multiplied together to predict courtship behaviour in male guppies. Could this be a general principle for how external and internal factors interact in other motivational systems and other species? Sadly, the biology of motivation is not this simple. In the case of guppy courtship we need to be very careful in interpreting the shape of the curves, because the measurement of the males' markings was based on a rank order as opposed to a true interval scale.

More generally, the relative role of internal and external factors and the precise way in which they interact to produce behaviour appear to vary extensively between different motivational systems. Most seem to require both, but there is a spectrum, with some motivated behaviours affected predominantly by internal stimuli and others primarily by external stimuli. Examples of the former include the way a rise in testosterone levels is sufficient to start singing behaviour in a male songbird such as a starling; the nest building of sows is another case in point. In contrast are those motivated behaviours affected predominantly by external stimuli. One example here would be explorative behaviour in mice, which is not seen at all when an animal is in its home cage, but is elicited by placing it in a novel environment; other examples include aggression and anti-predator behaviour.

A recent experiment with caged mink clearly demonstrates how external factors are more important for some motivational systems than for others. Warburton and Mason (2003) compared individual mink's motivation to reach four different resources in parallel (food, swimming water, toys and social contact). Animals had to push one of four weighted doors (depending on which resource they were motivated to access), and then travel along a tunnel $7.5 \mathrm{~m}$ long to reach the resource. This tunnel either doubled around to reach resources that were visible from the animal's home cage, and were thus visible when it pushed the weighted door ('cues treatment'); or it went behind an opaque screen to where the various resources were placed out of sight from the home cage ('no cues treatment'; see Fig. 4.3a). Whether or not cues were present when the mink chose to push the door affected their motivation to visit some of the resources. When their cues were not detectable from the home cage, mink visited toys and a conspecific less often than they did for food, but when cues were detectable they visited them as often. In contrast there was no effect of cues on visits to food or swimming water (see Fig. 4.3b). Furthermore, the minks' 'consumer surplus' (a measure of value or motivational importance) for toys, but no other resource, was significantly lower when the toys' cues were screened compared with when they were visible at the choice point. Thus the effect of external stimuli varies depending on which motivation you are dealing with.

The odd behavioural responses that we see in animals in some artificial situations can be very revealing about motivational mechanisms underlying behaviour. Animals deprived of the opportunity to perform behaviour patterns will sometimes show so-called 'vacuum activities', behaviour patterns occurring in the absence of the normal external eliciting stimuli. For example, hens housed in battery cages with wire floors will still go through the actions of dust bathing despite the absence of any suitable substrate. At the other end of the spectrum, external stimuli can sometimes alone be so powerful that they can trigger inappropriate behaviour. A herring gull will retrieve eggs that have rolled out of its nest but, when given a choice of two eggs, one of natural size and one $50 \%$ larger, it will preferentially retrieve the larger egg. The larger egg is a good example of what ethologists call a 'supernormal stimulus', because it is more effective 
(a) Apparatus
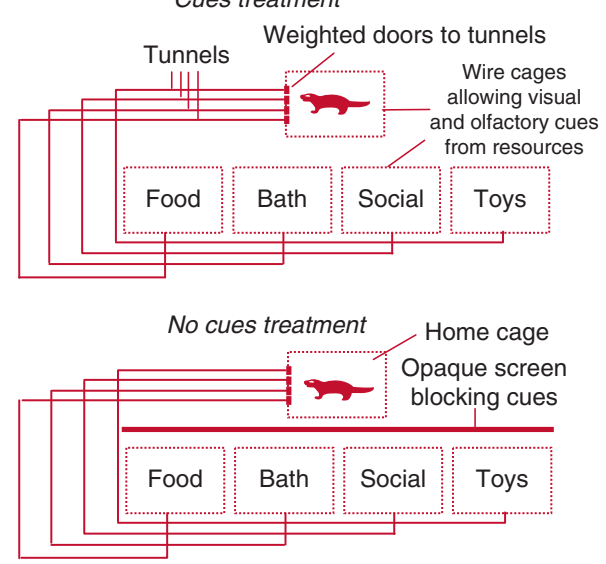

(b) Results

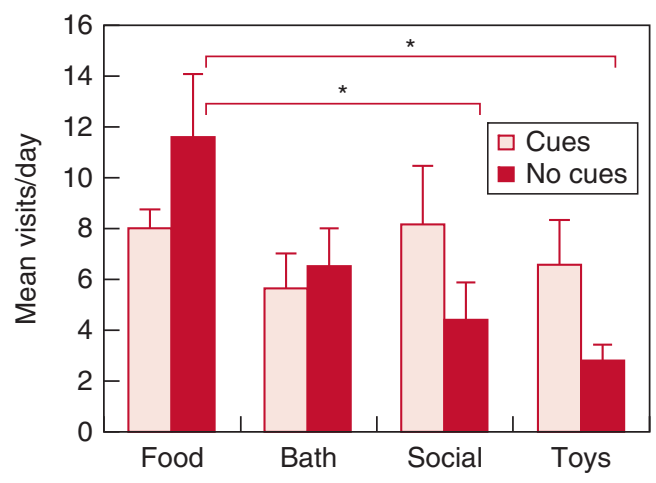

Fig. 4.3. An experiment to explore the effect of the availability of external cues on minks' use of four resources (food, swimming bath, access to social contact and toys). (a) The apparatus used in the two treatments of the experiment; in the 'no cues' treatment the screen blocks visual cues from the resources at the time the mink decides whether to work to access a resource; (b) the mean visits per day (one simple measure of motivation) made by the mink to each of the resources. Asterisks indicate significant differences (after Warburton and Mason, 2003, who also show further data on other indices of motivation).

at eliciting behaviour that the natural stimulus. Supernormal stimuli are likely to be very common in captive environments due to the high densities at which animals are housed, thus amplifying the normal cues provided by other animals in the environment.

\section{Regulatory processes in the motivational control of behaviour: negative feedback processes, hysteresis, positive feedback and switching costs}

Behaviour usually occurs in discrete bouts. For example, animals typically eat in 'meals', as opposed to nibbling constantly (lions can eat $30 \%$ of their own bodyweight in one sitting!) and, in the hen, dust bathing typically occurs in bouts of about 20 min that are performed every 2 days. Therefore, some motivational mechanism is needed to explain why a bout of behaviour continues as it does, and then ends. Negative feedback is the process whereby execution of a behaviour pattern reduces the motivation to perform it, and is important in limiting the length of bouts of many different behaviours. Negative feedback may come from the direct consequences of the behaviour. For example, the absorption of water into the body post-drinking causes a cascade of physiological consequences that reduce motivation to drink. Negative feedback may also come from the performance of a behaviour pattern per se. For example, the satiating effect of water injected into the body intravenously is not as great as water drunk by mouth. Similarly, mutant featherless hens will still go through the actions of dust bathing despite the fact that the behaviour can be having no effect on the condition of their (non-existent) plumage. This evidence supports the 
hypothesis that dust bathing has rewarding consequences for chickens above and beyond its direct hypothesized function in feather maintenance.

Whereas negative feedback is important in stopping animals from performing a behaviour pattern indefinitely, mechanisms are also necessary to make sure that animals persist at a behaviour for long enough to achieve its functional goal. A simple, competition model of motivation whereby an animal always immediately switches to the behaviour with the highest motivation suffers from the problem that it will leave animals constantly 'dithering' between, for example, tiny bites of food and tiny sips of water (see Fig. 4.4). A range of solutions has been suggested to deal with this problem, since real animals do not typically behave like this.

Delayed negative feedback (or 'hysteresis'), whereby it takes a period of time for negative feedback to reduce motivation, is one feature of motivational control that arrests dithering and gives bouts some stability. Furthermore, this is often aided by positive feedback which, although similar in effect, differs in that motivation actually increases after the start of a bout. One good example comes from careful studies of eating in mice by Wiepkema (1971). He analysed the 20-30 mini-bouts of eating that comprise each single meal a mouse eats. When mice are food deprived for $24 \mathrm{~h}$, minibouts increase in length (from about 8 to $13 \mathrm{~s}$ ) and the gaps between them decrease (from about 14 to $9 \mathrm{~s}$ ), illustrating an effect of deprivation. However, within each meal, and particularly at the start, mini-bouts initially also increased in length and occurred more closely together (thus, for example, the fifth mini-bout of a meal is longer than the first), nicely illustrating the effects of positive feedback. Later on in

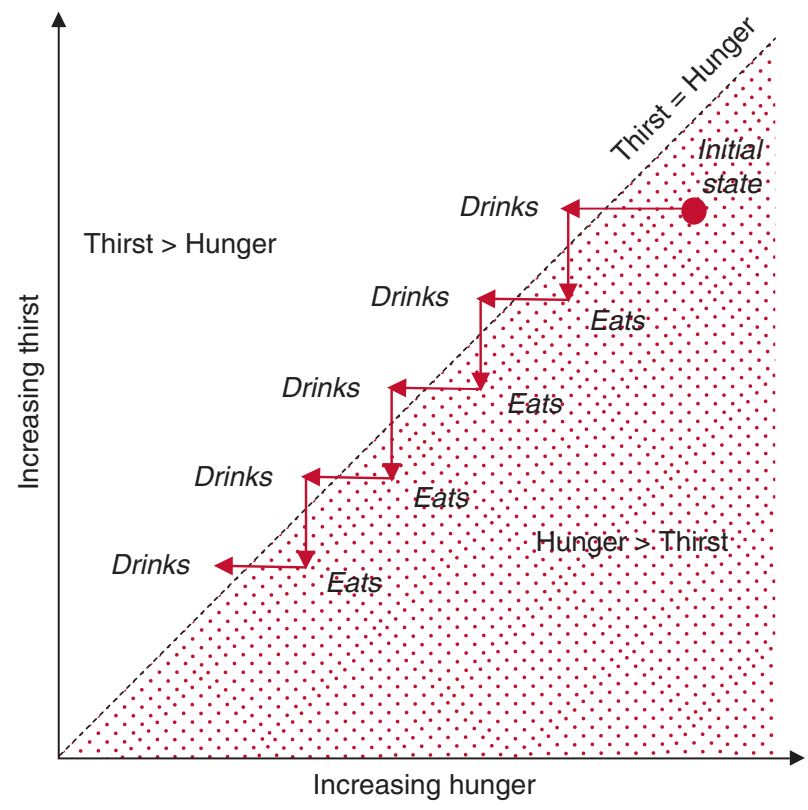

Fig. 4.4. Dithering between eating and drinking behaviour predicted by a simple competition theory of motivation. The animal is assumed to reduce its initially higher motivation, in this case hunger, until thirst is greater than hunger (i.e. the dotted line has been crossed), at which point it starts drinking. This model does not describe well what animals typically do in realistic situations (for explanations, see text) (modified from McFarland, 1985). 
the meal, the mini-bouts then decrease in duration again, illustrating the negative feedback that eventually terminates the meal.

Another factor affecting the length of behavioural bouts is the cost of switching to a different behaviour. Animals stick longer at a single type of behaviour (i) if it is costly in terms of time, energy or potential danger to switch to another; or (ii) perhaps because the motivation for (ii) has to build up to a higher level before the animal will be prepared to pay high costs to switch. A classic example of this phenomenon is provided by McFarland's (1971) study (described in McFarland, 1985) of switching between eating and drinking in doves. He showed that, if the birds had to negotiate a barrier to switch between these two behaviours, then they switched less often when the barrier was large than when it was small. It appears therefore, that animals take into account the cost of switching activities when decision making.

If the explanation for the delay in switching is that motivation has to build to a higher level before the animal is prepared to pay the switching cost, then this higher motivation should be reflected in the behaviour of the animal when it finally makes the switch. Data supporting this prediction are provided by an experiment in which mink were offered an array of different enrichments to interact with, but the costs of reaching each were increased by weighting the doors that the mink had to push to reach them. Increased costs resulted in two main changes in behaviour: first, as with McFarland's doves, the animals switched between enrichments less often, using each enrichment for longer in each bout; secondly, when they eventually paid the cost to use a new enrichment, they had a shorter latency to interact with it after pushing open the door. This latter result illustrates that, when the costs of switching were higher, a higher motivation had built up before the animal chose to pay the cost of switching (Cooper and Mason, 2000).

\section{Behavioural sequencing: appetitive and consummatory behaviour and feed-forward processes}

Bouts of motivated behaviour rarely comprise the exact same motor sequence repeated again and again, but usually have some kind of internal structure to them. For example, a bout of dust bathing in a chicken starts with the bird scratching and bill-raking at the substrate. Next it erects its feathers and squats in the substrate and performs a sequence of vigorous dust bathing behaviours. This phase is followed by the bird flattening its feathers and lying on its side. Finally it stands up and shakes the substrate from its plumage before switching to another activity. All of these phases within the sequence appear to be motivated by a single state.

A distinction is often made between 'appetitive' and 'consummatory' phases within a behavioural sequence. The appetitive phase comes first, and comprises active, flexible, searching behaviours; appetitive behaviour can even include novel responses that the animal learns, e.g. lever pressing. Appetitive behaviour may appear quite similar even when motivated by quite different states. For example, the appetitive phases of food search, mate search and nesting site search are all superficially quite similar both within and between species. Appetitive behaviour is followed by consummatory behaviour that is more stereotyped, unlearnt, species-typical and motivation-typical. Examples include the specific movements used in foraging (e.g. cows and giraffes tongue-twirl, sheep and goats bite, pigeons peck); drinking 
(e.g. cats and dogs lap, pigs and humans suck, starlings dip-and-tilt); and mating (e.g. lordosis by female quadrapeds, thrusting by males). The term 'fixed action pattern' is often used for the stereotyped, species-typical forms involved. Analysing fixed action patterns can be a useful way of identifying the motivational bases of abnormal behaviour, as we will see in Section 4.4.

In addition to the predictability within a sequence of motivated behaviour, transitions between one type of motivated behaviour and another are also often rather predictable. Thus, often one behaviour pattern follows another: drinking follows eating (in, e.g. rats and humans) and may be followed by thermoregulatory behaviour (if drinking reduces core body temperature); grooming follows eating in cats; and, preening follows water bathing in birds. Such adaptive sequences of behaviour may be partially the product of 'feed-forward' mechanisms. Traditionally, feeding and drinking behaviour were thought to be controlled by feedback mechanisms that tracked the levels of physiological indices of hunger and thirst (see above). However, eating typically motivates subsequent drinking even before the osmotic consequences of food intake have commenced, suggesting that feed-forward mechanisms may be more important in the control of feeding and drinking than previously thought. In at least some instances, such feed-forward effects are the product of past experience and learning (e.g. Ramsay et al., 1996).

\subsection{Models of Motivation}

In order to conceptualize how internal and external factors are integrated, and how negative feedback, positive feedback and other processes occur, ethologists have modelled motivational states in a number of different ways. As we shall see, different models concentrate on explaining different aspects of the observed behavioural phenomena that we have described above.

\section{The psychohydraulic model}

One early model of motivation was that of Lorenz (1950, described in McFarland, 1985). His 'psychohydraulic model' likens behavioural control to a cistern of water that can overflow via a valve, this overflow representing the performance of behaviour (see Fig. 4.5). The cistern steadily fills from an in-pipe, representing increasing levels of internal stimuli when a behaviour is not performed. Overflow via the valve occurs if a combination of the pressure due to the build-up of water in the cistern (representing the build-up of internal stimuli) and weights pulling on the valve (representing the influence of external stimuli) reaches a critical threshold. Flow ceases again once the fluid level in the cistern, and hence the internal pressure, have decreased.

This model admirably represents the ways that internal and external factors combine to determine motivation; that behaviour patterns occur in predictable sequences; and that several aspects of behaviour change with increased motivational state, including duration, rate and the types of behaviour patterns produced. However, one criticism of this model is that it implies that the motivation always remains high unless behaviour is performed, because overflow via the valve is the only way for the cistern to empty. While this may be appropriate for certain behaviours (e.g. eating and drinking), it clearly is not the case for others: as we have already discussed, 


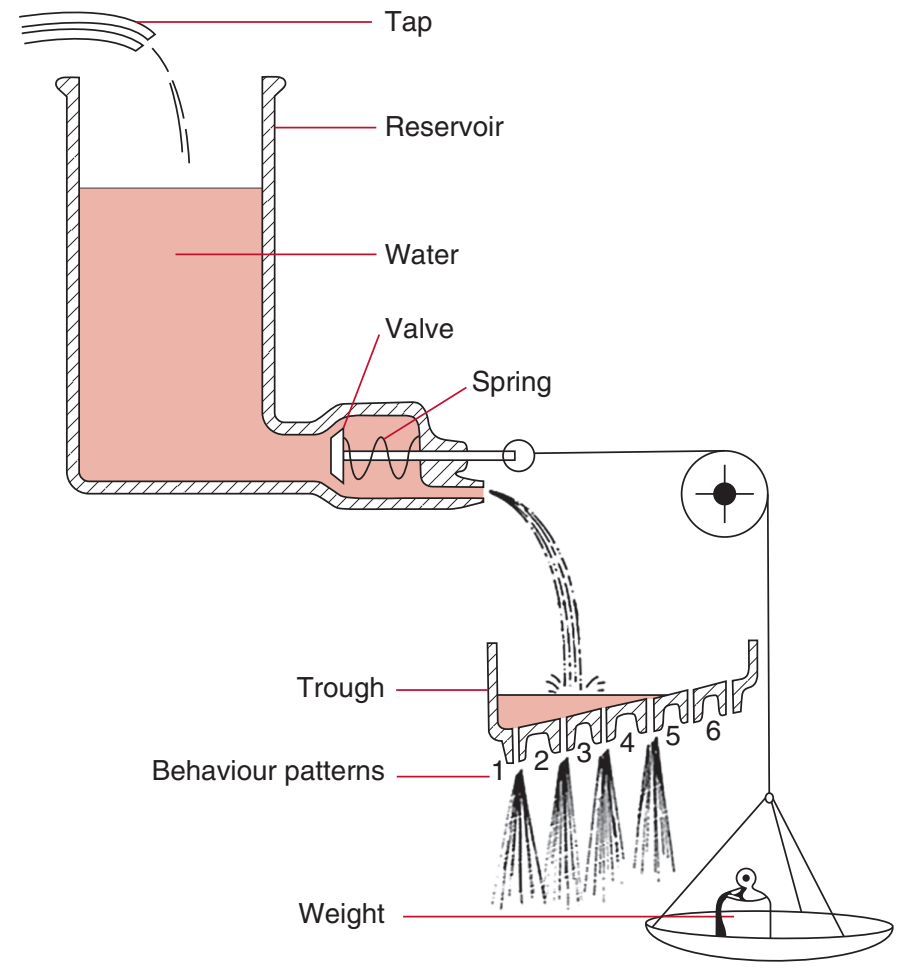

Fig. 4.5. Lorenz's psychohydraulic model of motivation. Behaviour occurs when water exits the reservoir into the trough. The spring holds the valve shut until the valve is opened by a combination of the pressure of water that has built up in the reservoir (i.e. internal state) and the weight in the pan (i.e. external stimuli). The behaviour pattern displayed (1-6) depends on the level of water in the trough, with higher patterns requiring higher levels of water (modified from Lorenz, 1950 in McFarland, 1985).

internal factors seem unimportant for some behaviours. In the absence of rivals, for instance, an animal will not become more and more likely to fight, and likewise if predators are absent, an animal will not become more likely to hide or make alarm calls. Thus for many behaviours, if the goal of a behaviour exists already, the motivation to perform that behaviour will remain low.

\section{The Sollwert-Istwert model}

That same year, von Holst and Mittelstaedt (1950, described in Mason et al., 1997) and colleagues developed an alternative model that did not assume an inevitable build-up of internal causal factors in the absence of behavioural expression. Their 'Sollwert-Istwert' model conceptualizes motivational systems as acting more like a thermostat, causing behaviour that brings an animal's state (its 'Istwert' - literally, 'the way the world is') closer to a desired end point (the 'Sollwert' - 'the way the world should be'). The greater the discrepancy between Sollwert and Istwert, the stronger the motivation to perform the behaviour. 
The important difference between this and the psychohydraulic model is that it emphasizes that motivation may be extremely low if the end point of the behaviour is already in existence. However, a similarity between the two models is that negative feedback is the key means of behavioural control. Since this is too simple to account for how real animals partition their time between different activities, the SollwertIstwert model was used as the starting point for more sophisticated representations of the control and sequencing of behaviour, considered next.

\section{Systems models}

So-called 'control systems' models of motivation are based on the assumption that we can draw analogies between the mechanisms of living and non-living systems (McFarland, 1971, described in McFarland, 1985). These models incorporate means of control other than negative feedback so that, for example, motivation can increase in anticipation of a discrepancy between Sollwert and Istwert (i.e. a feed-forward process), rather than always reacting to discrepancies after the event. These models represent behaviour using systems diagrams or control theory from engineering (see Fig. 4.6). In contrast to the models above, they can represent behavioural sequences (e.g. appetitive and consummatory phases) and also these often incorporate stages of feed-forward, hysteresis and positive feedback. These models also incorporate something valuable from the psychohydraulic model, i.e. the performance of behaviour per se can, at times, be important in reducing motivation. Furthermore, Hughes and Duncan (1988) did a valuable job in refining these models and used them to discuss 'ethological needs' and abnormal behaviour in captive animals, topics we return to in Section 4.4.

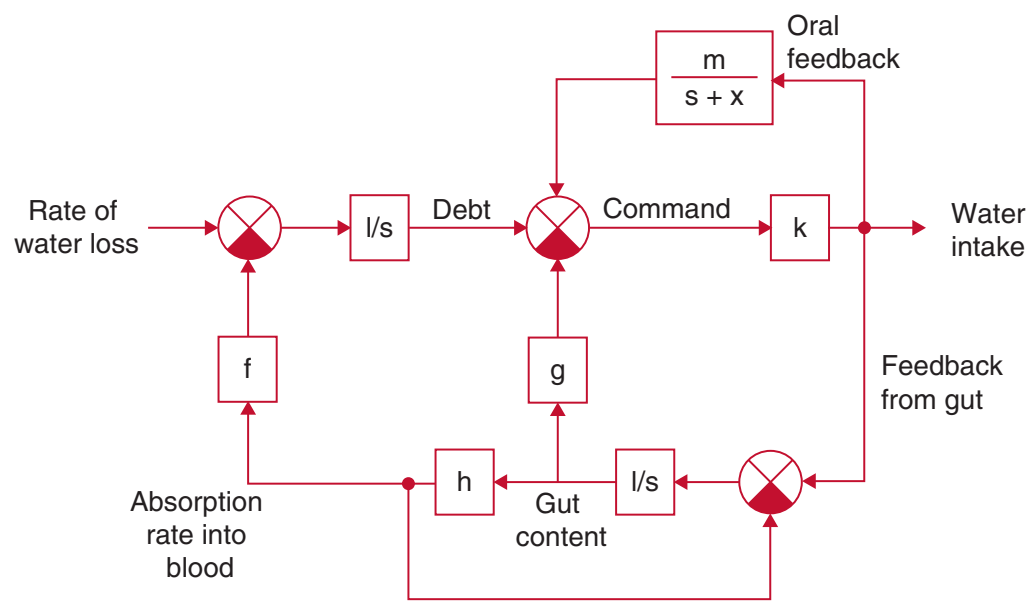

Fig. 4.6. A control systems model of drinking in doves. In this model oral stimulation during drinking provides positive feedback, and gut factors provide negative feedback. The circles represent points in the control system at which different variables are assumed to be summed up, and a black quadrant in one of these circles changes the sign of an input. The letters within the boxes correspond to parameters of the various component mechanisms (modified from McFarland and McFarland, 1968 in McFarland. 1985). 


\section{State-space models}

The next generation of motivational models focused in more detail on the interactions between behaviour patterns and the rules animals follow when choosing between different activities. State-space models (McFarland and Sibly, 1975, described in Barnard, 2004) represent an animal's motivational state as an $\mathrm{n}$-dimensional vector resulting from the interaction between internal and external factors. This motivational state is the state value of all the causal factors influencing a set of functionally related behaviour patterns. Each motivational state competes with others to control the animal's behaviour - the one expressed behaviourally being the one in which the combination of causal factors is the highest. Thus, this model emphasizes how what an animal does depends on what other motivations are high, that in turn might be affected by what else there is to do in the environment.

\section{A real example: a motivational model of dust bathing}

In this chapter we have described several features of dust bathing in hens: it occurs in discrete bouts of about 20 min duration, most often in the middle of the day. Hogan and Van Boxel (1993) suggested a simple motivational model of dust bathing that captures these basic characteristics. They began by assuming a simple Lorenzian process whereby an internal factor for dust bathing builds up over time, but dissipates rapidly during dust bathing bouts (see Figs 4.7 and 4.8). The level of the factor

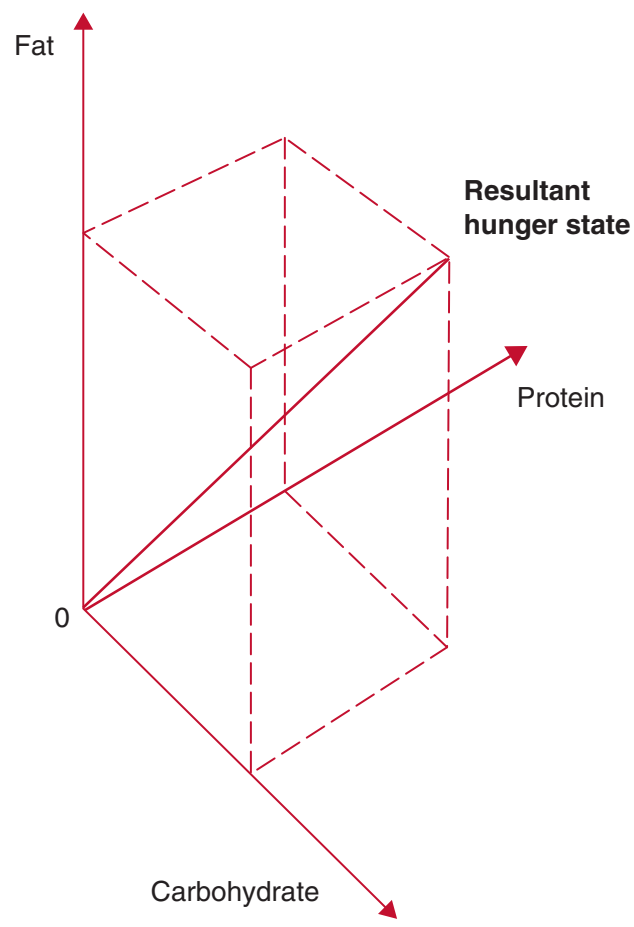

Fig. 4.7. The motivational state of hunger portrayed in a three-dimensional physiological space (modified from McFarland and Sibly, 1972 in McFarland,1985). 


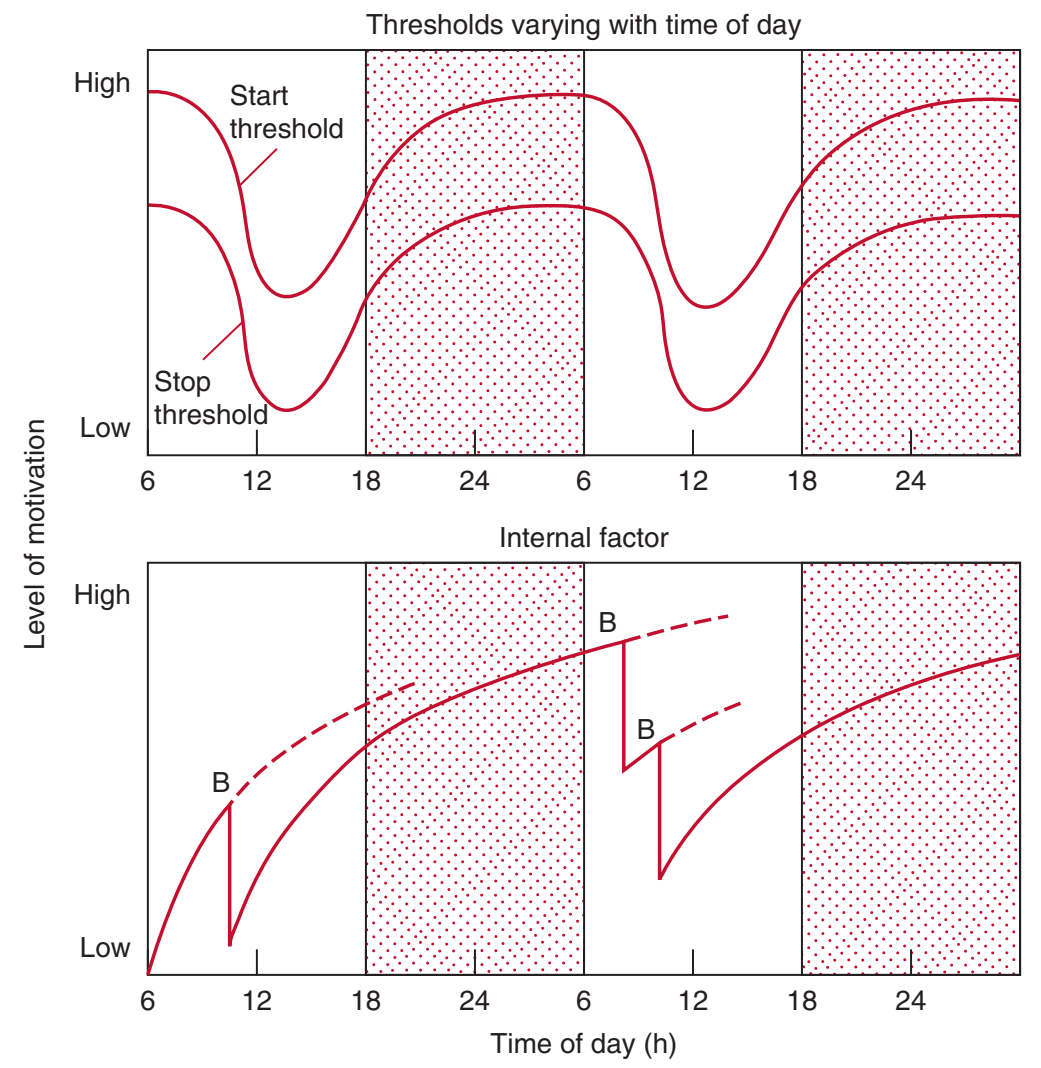

Fig. 4.8. A motivational model of dust bathing. The lower panel shows the internal factor for dust bathing that is assumed to increase over time in a decelerating manner, and to decrease rapidly when a bout of dust bathing occurs (B). The upper panel shows the parallel upper and lower thresholds assumed to start and stop dust bathing, respectively. Dust bathing starts when the level of the internal factor exceeds the start threshold, and stops when it falls below the lower threshold. External stimuli such as light, heat and also qualities of the substrate are assumed to affect the thresholds (modified from Hogan and Van Boxel, 1993).

necessary for dust bathing to occur was assumed to depend on a threshold that is high in the morning but much lower in the middle of the day. If dust bathing continued until the internal factor returned to zero then the model would predict dust bathing bouts to be longer in the morning than at midday (which is not observed), therefore it was also necessary to assume a lower stopping threshold that varies in parallel with the starting threshold.

In ethology a good model of any kind will not only capture facts that we already know to be true about the control of a behaviour pattern, but will also make novel predictions that we can test. If the circadian variation in the height of the threshold is due to changes in light and temperature (a reasonable assumption to make), then we would predict that applying additional light and heat should shift the timing of dust bathing, but not the total amount performed. Hogan and Van Boxel (1993) were 
able to confirm these predictions in a study of Burmese red junglefowl. However, subsequently, other ethologists have argued that the above model does not capture some of the data on dust bathing, and have suggested alternative approaches (for more on this interesting topic see Olsson and Keeling, 2005).

\subsection{Motivation and Applied Ethology}

There are four key reasons for those interested in domestic animal behaviour to understand motivation, and we end our chapter with these.

\section{Unfulfilled motivations and poor animal welfare}

The first reason to understand motivation is related to animal welfare, and arises from the links between motivation and emotion. Emotions can be thought of as the states in an animal that are elicited by reward or punishment, whereas motivations can be thought of as states in which a reward is being sought or a punisher avoided (Rolls, 2005). Following these definitions, positive and negative emotions play an important role in the motivation of behaviours. In humans, for example, the affective (i.e. emotional) pleasure associated with drinking increases with motivation to drink, and the effective pleasure associated with touching something warm increases with motivation to be warm. This link between motivation and emotion led Dawkins (1990) to suggest that assessing motivational priorities was crucial for maximizing the welfare of animals kept in captivity: if captive animals prove highly motivated for resources they are denied in their typical housing conditions, then we should perhaps assume poor welfare due to negative emotions.

All too often, captive animals have insufficient resources or opportunities for homeostasis, e.g. hunger (pregnant sows, broiler breeders, dairy calves), excessive cold/heat (mink underfed during the winter, cattle in shadeless feedlots, pigs transported in trucks) and other threats to homeostasis. When presented with opportunities to restore homeostasis, these animals show themselves highly motivated. For example, broiler breeds that are typically fed less than one-third of the ration they would choose to consume will work hard to obtain extra food from an operant schedule. Animals are not only motivated to restore homeostasis but, when asked experimentally - if they will work for the chance to perform naturalistic activities, often answer 'yes', even when these behaviours offer no nutritional or other physiological benefit.

Thus, pregnant sows will work to build nests, as will hens about to lay. Mothers will often work for infant cues and, in some animals, same-age conspecifics are also valued. For example, isolated primates, rats and red fox vixens will all work hard for social company. Elements of natural foraging behaviours also seem highly motivated, even when they do not result in the actual acquisition of food. Thus rats, mice and many other animals will perform operant responses for the chance to run in a running wheel; the naturally semi-aquatic mink will work to swim; and pigs will work for the chance to root in bare earth or chopped straw. There are many other cases - examples that are poignant given that these animals are often denied these natural behaviours in captivity. 
Of course, to be sure that such data really demonstrate poor welfare in captivity, we need to check that these measured motivations are not simply elicited by cues from the resources to which these animals are exposed during these tests. However, in at least some of these examples (e.g. nest building for sows, social isolation for rats and primates and perhaps also swimming for at least some genetic subtypes of mink), there are good additional stress-related data (see Chapter 7) to show that preventing these natural behaviours in captivity is a real cause for concern. Natural behaviours, the prevention of which causes suffering, are termed 'ethological needs'.

\section{Understanding stereotypic behaviour}

The second reason to investigate domestic animals' motivations is that this can help us understand why abnormal behaviour patterns like stereotypic behaviours are so common (see Chapter 7). Stereotypic behaviours - abnormal repetitive behaviours with no apparent goal or function - are prevalent in captive animals: over 85 million perform them worldwide and, in some populations (e.g. zoo-housed giraffes and single-housed laboratory primates), they are nearly ubiquitous.

Ethological explanations for these behaviours have focused on frustration, since they often seem to arise from attempts to perform highly motivated normal behaviour patterns; in many instances, depriving animals of chances to perform behaviour by removing important resources induces or exacerbates stereotypies. Thus caged wildcaught birds typically show more route tracing than captive-bred birds, possibly because they are trying to escape; premature maternal separation typically induces similar stereotypic attempts to escape, or instead to suckle, in young mammals; and removing or delaying expected food rewards elevates stereotypic pacing, weaving and oral behaviours in captive pigs and carnivores.

The first biologists to notice and record these behaviours typically explained them as caused by internal states of deprivation (stemming from the loss of the mother, say, or from insufficient food), perhaps combined with strong eliciting stimuli (the sight of other conspecifics or the sounds and smells of other nearby animals being fed), leading to sustained high motivations that the subject cannot reduce: it cannot perform the activity that would result in negative feedback. Powerless to effect real change, the animal is able only repeatedly to attempt to perform a substitute for the relevant behaviour, or try and try again to escape the frustrating situation (see, e.g. Hughes and Duncan, 1988). The lack of motivational competition typical of barren captive conditions was also assumed to contribute to the prolongation of bouts of stereotypic behaviour.

Some of these motivational hypotheses have recently been tested experimentally. Important work in the 1980s on hunger and stereotypic oral activities in pregnant sows has already been extensively reviewed elsewhere. Here we present some newer and perhaps less familiar examples. For example, Wiedenmayer (1997) observing the stereotypic digging of caged gerbils, reasoned that, in the wild, the stimulus that would bring digging to a close would be a tunnel leading to a nesting chamber. He tested this idea in the laboratory and found that gerbils that had been raised with extra space, sand to dig in or a simple plastic nesting chamber still showed the abnormal behaviour but animals raised with a nesting chamber reached via a plastic tunnel did not. Nevison and colleagues (1998) similarly manipulated external stimuli to test the idea 
that bar mouthing by laboratory mice derives from repeated escape attempts. They found that, if a door in the cage-lid was regularly opened and mice sometimes allowed to exit from it, their bar chewing became directed to that site; on the other hand if clear plexiglass was placed over a region of the cage-lid, to prevent odour cues from entering at that point, mice would move their bar mouthing elsewhere.

More recent work still has focused on feather pecking by hens, demonstrating that it derives from a very specific form of redirected foraging (rather than from redirected dust bathing pecks, as had been suggested in the 1980s). HarlanderMatauschek and colleagues (2007) discovered that individual hens who display a lot of feather pecking are actually highly motivated to ingest feathers: offered bowls of food, shaving and feathers, they approach the feathers more rapidly, stay at the feather-bowls for longer and ingest more feathers than non-feather-pecking individuals. They will even perform an operant to gain feathers to eat. Meanwhile other researchers (Dixon et al., 2008) examined the 'fixed action patterns' involved in feather pecking: their careful quantitative comparison of the morphology of feather pecks with foraging pecks, dust bathing pecks, drinking pecks and exploratory pecks showed that foraging and feather pecking involved near-identical fixed action patterns.

These explanations, in terms of specific motivational frustrations, seem likely to explain the form and timing of many abnormal behaviours. However, they do not seem to be the whole story. There is growing evidence that these strange behaviours are exacerbated by impairments within the brain - induced by stress and/or by abnormal development - that predispose animals to pathological levels of inappropriate repetition. Some of these changes are quite independent of motivational affects, and simply add to them, or enhance them into the clockwork-like behaviours that are sadly so common (see Chapter 7). Some, however, may involve broader motivational abnormalities: generalised tendencies to respond, inappropriately excessively, to potential rewards perhaps related to the tendencies involved in human compulsions and addictions (see Chapter 7). This is an exciting area for future research.

\section{Reward and motivation in learning and training}

The third reason again relates to the links between motivation and emotion: that understanding motivation can increase the ease with which we can train animals to perform behaviour patterns that we want.

Motivation and learning are intimately linked: the opportunity to perform highly motivated behaviours is a powerful 'reinforcer' in learning - far more so than the opportunity to perform behaviours that are not highly motivated. Thus in choosing, say, how to use food treats to reward desired behaviours during training, we need to understand that these will be effective only if the animal is motivated to obtain them. The use of unpalatable food rewards, or the animals being sated thanks to just having eaten a large meal, will therefore impede training, while in contrast the use of highly palatable treats, or prior moderate food deprivation, combined with the use of small treats that do not sate the animal, will all facilitate training by helping to ensure that the animal is sufficiently motivated to learn the behaviours we desire so that it can obtain the food rewards. This can have animal welfare implications too; rather than food-depriving rats or mice to get them to run tasks in the 
laboratory, researchers can choose to use highly palatable sweet breakfast cereals as rewards. Similarly, non-deprived hens and starlings will work for highly desirable mealworms.

\section{Encouraging species-typical behaviour}

The fourth and final reason is similar to the previous three in that, by appreciating how internal and external factors combine, in an unlearnt way, to motivate behaviour, it can help us 'persuade' the animals we keep to behave as we humans want them to.

For example, if we appreciate how dam endocrine state and lamb odour combine to motivate maternal care in sheep, we can use this information to devise the best ways to manipulate ewes so that they will accept orphan lambs (by timing our attempts appropriately relative to parturition; and by modifying olfactory cues from the orphaned lamb). We can use our knowledge that highly motivated animals will be less choosy about how to their satisfy their motivations, to persuade sexually aroused stallions to ejaculate into an artificial mare for semen collection (by first using a 'teaser' mare, in oestrus, to arouse the stallion); or similarly to persuade females to mate with sub-standard males. Conversely, we can appreciate that if internal factors motivating, say eating, are low, we will have to use particularly palatable foodstuffs - i.e. treats that present particularly powerful eliciting external stimuli - if we want to persuade ill or frightened animals in our care to eat.

\subsection{Conclusions and Links to Other Chapters}

In this chapter we have provided a brief introduction to the data and theories relating to the concept of motivation, and have discussed these in the context of some of the current challenges in applied ethology. We would like to end by returning to Tinbergen's four questions and emphasizing the strengths of the ethological approach. Although the study of motivation focuses on questions of proximate causation, in trying to understand the motivation for a particular behaviour pattern it will often be important to consider Tinbergen's other three questions (see Box 4.1). The causal mechanisms responsible for a behaviour pattern are often affected by the developmental experience of an animal, and thus a good understanding of the ontogeny of a behaviour pattern could be crucial in understanding why a captive animal is behaving abnormally. The causal mechanisms responsible for a behaviour pattern have evolved in order to motivate an animal to perform behaviour patterns that will optimize its survival and reproduction. Therefore, understanding the ultimate goal and survival value of a behaviour pattern, and its phylogenetic roots, is also likely to be important in understanding how it is controlled. This is particularly important when solving problems in applied ethology because, as we have seen, the unfulfilled ethological needs and abnormal behaviour patterns of captive animals may often have their origins in adaptive behaviour.

\section{Acknowledgements}

Thanks to Laura Dixon and Naomi Latham for some useful examples, and to David Fraser for Fig. 4.1. 


\section{References}

Barnard, C. (2004) Animal Behaviour: Mechanism, Development, Function and Evolution. Pearson, Harlow, UK.

Berridge, K.C. (2004) Motivation concepts in behavioral neuroscience. Physiology and Behaviour 81, 179-209.

Boulton, M.I., Wickens, A., Brown, D., Goode, J.A. and Gilbert, C.L. (1997) Prostaglandin F2 alpha-induced nest-building in pseudopregnant pigs. 1. Effects of environment on behaviour and cortisol secretion. Physiology and Behaviour 62, 1071-1078.

Cooper, J.J. and Mason, G.J. (2000) Increasing costs of access to resources cause rescheduling of behaviour in American mink Mustela vison: implications for the assessment of behavioural priorities. Applied Animal Behavior Science 66, 135-151.

Dawkins, M.S. (1990) From an animal's point of view: motivation, fitness and animal welfare. Behavioural and Brain Sciences 13, 1-61.

Dixon, L, Duncan, I.J.H. and Mason, G.J. (2008) What's in a peck? Using fixed action patterns to identify the motivation behind feather-pecking. Animal Behaviour 76, 1035-1042.

Harlander-Matauschek, A., Benda, I., Lavetti, C., Djukic, M. and Bessei, W. (2007) The relative preferences for wood shavings or feathers in high and low feather pecking birds. Applied Animal Behaviour Science 107, 78-87.

Hogan J.A. and Van Boxel, F. (1993) Causal factors controlling dust bathing in Burmese red junglefowl: some results and a model. Animal Behaviour 46, 627-635.

Hughes, B.O., and Duncan, I.J.H. (1988) The notion of ethological 'need', models of motivation and animal welfare. Animal Behaviour 36, 1696-1707.

Mason, G., Cooper, J. and Garner, J. (1997) Models of motivational decision-making and how they affect the experimental assessment of motivational priorities. In: Forbes, J.M., Lawrence, T.L.J., Rodway, R.G., and Varley, M.A. (eds) Animal Choices. Occasional Publication No. 20, British Society of Animal Science, Penicuik, UK, pp. 9-17.

McFarland, D. (1985) Animal Behaviour. Longman Scientific and Technical, Harlow, UK.

Nevison, C.M., Hurst, J.L. et al. (1999) Why do male ICR(CD-1) mice perform bar-related (stereotypic) behaviour? Behavioural Processes 47(2), 95-111.

Olsson, I.A.S. and Keeling, L.J. (2005) Why in earth? Dust bathing behaviour in jungle and domestic fowl reviewed from a Tinbergian and animal welfare perspective. Applied Animal Behaviour Science 93, 259-282.

Ramsay, D.S., Seeley, R.J., Bolles, R.C., and Woods, S.C. (1996) Ingestive homeostasis: the primacy of learning. In: Capaldi, E.D. (ed.) Why We Eat What We Eat. American Psychological Association, Washington, DC.

Rolls, E.T. (2005) Emotion Explained. Oxford University Press, Oxford, UK.

Warburton, H. and Mason, G. (2003) Is out of sight, out of mind? The effects of resource cues on motivation in mink, Mustela vison. Animal Behaviour 65, 755-762.

Wiedenmayer, C. (1997) Causation of the ontogenetic development of stereotypic digging in gerbils. Animal Behaviour 53, 461-470.

Wiepkema, P.R. (1971) Positive feedback at work during feeding. Behaviour 39, $2-4$. 